\section{Efficacy of interdental calibrated brushes on bleeding reduction in adults: a 3-month randomized controlled clinical trial}

\author{
Bourgeois D, Saliasi I, Llodra JC, Bravo M, Viennot S, Carrouel F. Efficacy of \\ interdental calibrated brushes on bleeding reduction in adults: a 3-month randomized \\ controlled clinical trial.
}

Eur J Oral Sci 2016; 00: 000-000. (C) 2016 Eur J Oral Sci

This study investigated the effect of interdental brushes on the reduction of interproximal bleeding in adults without periodontal disease. Forty-six adults were enrolled in a 3-month, observer-blinded, parallel-group randomized control trial. The test group used a standard manual toothbrush twice daily and an interdental brush daily. The control group used a standard manual toothbrush. At each visit, a calibrated colorimetric probe was used in all interdental spaces to determine the appropriate size of the interdental brush required, the brush of the corresponding size was introduced into the interproximal space, and the presence of bleeding was recorded. The outcome was the frequency of bleeding after application of the appropriate interdental brush. All participants were evaluated $1 \mathrm{wk}, 1 \mathrm{month}$, and 3 months after the baseline visit. The overall preventive fraction with respect to bleeding frequency was $46 \%$ at $1 \mathrm{wk}$ and $72 \%$ at 3 months. More bleeding reduction was observed in anterior sites than in posterior sites $(80 \%$ vs. $69 \%$, respectively). Participants with low baseline bleeding frequency presented with less bleeding $(\mathrm{OR}=2.3)$. This study shows that daily use of calibrated interdental brushes reduces interdental bleeding. These findings may support interdental cleaning as an effective means to help individuals maintain and/or achieve optimal oral health.

\author{
Denis Bourgeois ${ }^{1, *}$, Ina Saliasi ${ }^{1, *}$, \\ Juan Carlos Llodra ${ }^{1}$, \\ Manuel Bravo², Stéphane \\ Viennot $^{1, *}$, Florence Carrouel ${ }^{3, *}$ \\ 'Laboratory 'Course, Health and Systemic', \\ EA4129, University Lyon 1, Lyon, France; \\ ${ }^{2}$ Department of Preventive and Community \\ Health, Faculty of Dental Medicine, University \\ of Granada, Granada, Spain; ${ }^{3}$ Institute of \\ Functional Genomics of Lyon, UMR5242, \\ ENS Lyon, University Lyon 1, Lyon, France \\ ${ }^{*}$ These authors contributed equally to this work.
}

Denis Bourgeois, Laboratory 'Course, Health and Systemic', EA4129, University Lyon 1, 11 rue Paradin, 69372 F. Lyon, France

E-mail: denis.bourgeois@univ-lyon1.fr

Key words: gingival bleeding; interdental biofilm; interdental brushes; oral prophylaxis

Accepted for publication August 2016
Disruption of the oral biofilm in interproximal sites by mechanical methods remains one of the best options for preventing periodontal disease (1). Well-designed randomized control trials (RCTs) are required to provide evidence supporting the clinical practice of the new hypothesis that interdental cleaning should become an established part of daily oral hygiene for the reduction of interproximal plaque in all populations. Originally, dental professionals recommended that patients with large embrasure spaces between their teeth should use interdental brushes (IDBs) $(2,3)$, mainly to prevent periodontal destruction. However, with the availability of a greater range of IDBs calibrated in size and crosssectional diameter, IDBs have become a potentially suitable alternative to dental floss for healthy patients who have interdental papillae that fill the interdental space $(4,5)$.

Oral hygiene instruction (OHI), which includes instruction in toothbrushing and interdental cleaning, is effective in disturbing oral biofilm and preventing periodontal diseases, such as gingivitis and periodontitis
(6). Studies have evaluated the effect of OHI on clinical and immunological parameters and on the microbiological profiles of periodontal maintenance participants, but no studies have evaluated its role in the management of gingivitis (7). It is unclear whether the daily use of IDBs is effective (3) because only low-quality evidence for IDB-related gingivitis reduction exists. The effectiveness of interdental/interspace brushes in addition to standard toothbrushes is not supported by clinical investigations. However, there are conflicting study results regarding the efficacy of IDBs in reducing the clinical parameters of gingival inflammation (8). There is also a lack of consensus regarding whether IDBs are only suitable for patients with moderate to severe attachment loss and open embrasures or are also a suitable aid for preventing gingivitis in healthy patients who have sufficient interdental space to accommodate them $(9,10)$.

The purpose of this study was to determine whether mechanical cleaning with IDBs, combined with the use of a manual toothbrush, is more effective than 
mechanical cleaning with a manual toothbrush alone in reducing interproximal bleeding in the gums of adults with gingivitis.

The study hypothesis was that the IDB is an effective alternative for reducing bleeding in the gums of individuals with gingivitis, allowing them to maintain and/or achieve optimal oral health and encouraging daily home use of IDBs.

\section{Material and methods}

The guidelines of the CONSORT Statement were followed in this clinical trial (11). The protocol was declared of public interest by the National Ethics Committee and was approved by the National Commission of Informatics and Liberties, France. It was performed in accordance with the Code of Ethics established by the Declaration of Helsinki. The study was performed according to the guidelines of Good Clinical Practice. Before participation, all participants received full oral and written information on the study aims and signed a written consent form.

The workflow design, summarized in Fig. 1, was a 3-month, observer-blinded, single-centre, stratified, parallel-group, RCT with patients allocated (1:1) to the following two groups: test group (manual toothbrush and interdental calibrated brushes); and control group (manual toothbrush).

Forty-six Caucasian adults, diagnosed as periodontally healthy, were recruited between September 2014 and December 2014 from a pool of first-time volunteers who were referred to the Department of Public Health of the Faculty of Oral Medicine at the University of Lyon (UCBL), France. Participants were included if they: (i) were $18-25 \mathrm{yr}$ of age; (ii) had at least 20 natural recordable teeth, including third molars; (iii) had no signs of clinical periodontitis; (iv) had no significant dental anomalies or prosthetic restorations or interproximal caries;

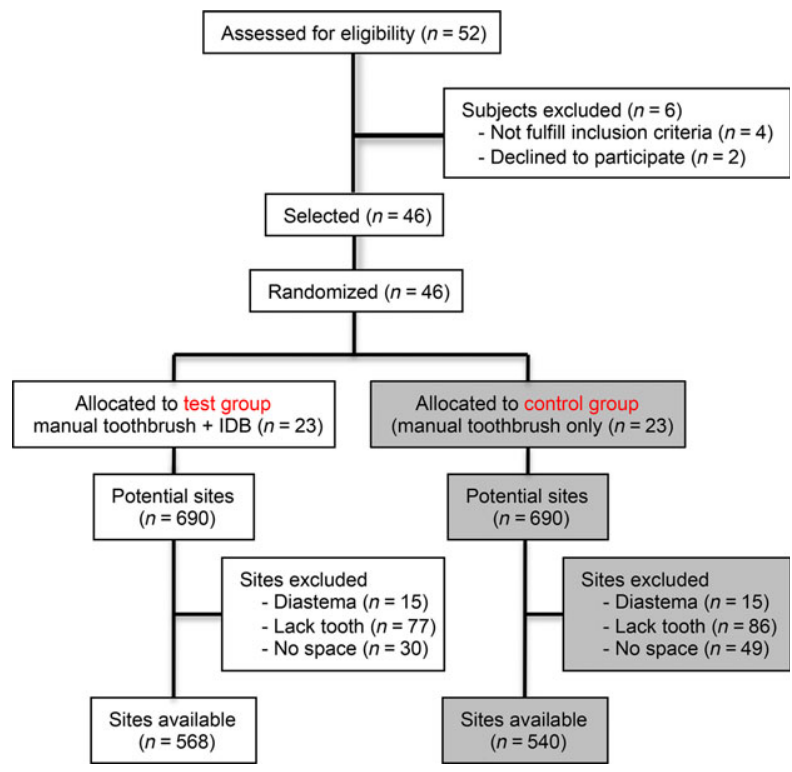

Fig. 1. Flow chart of the study design. IDB, interdental brush. (v) reported brushing teeth at least twice per day; (vi) had no health condition that required antibiotic prophylaxis before interproximal probing; (vii) had IDB dexterity; and (viii) lacked experience with interdental cleaning and were willing to undergo four study visits.

Exclusion criteria comprised: (i) teeth missing as a result of periodontal disease; (ii) any other concomitant systemic disorder; (iii) diseases affecting the immune system; (iv) receiving medication, such as anti-platelet or anti-coagulant agents; (v) pregnancy or breastfeeding; (vi) professional prophylaxis within $4 \mathrm{wk}$ before the baseline examination; (vii) history of periodontal disease or treatment; (viii) use of over-the-counter mouthwash; and/or (ix) undergoing a course of dental or orthodontic treatment. The use of antibiotics during the study period led to exclusion.

All participants met the criteria described by the American Academy of Periodontology (12), with some modifications (13). The healthy participants presented $\leq 10 \%$ of sites with bleeding on probing (BOP) after $30 \mathrm{~s}$ and/or overt gingival redness, and had no pocket depth of $>3 \mathrm{~mm}$ and no clinical attachment loss of $>2 \mathrm{~mm}$.

\section{Procedure}

Calculation of sample size (sites) was performed using Sample Power 2.0 (SPSS, Chicago, IL, USA). In the absence of exact values provided by theory or experience in this specific area, the $t$-test for independent groups was used to detect a power of $80 \%$ and $5 \%$ alpha error for an estimated Cohen's d of 0.5 - a medium effect size according to Cohen's scale (14) - in bleeding after IDB use. We needed 128 a-priori sites (64 test and 64 control). After considering a design effect (owing to sites being clustered within participants) of 5.0 (estimated from 20 pilot participants from this study) in estimating the bleeding percentage, the sample size increased to 640 sites $(=128 \times 5)$. Furthermore, after considering an estimated $70 \%$ of the sites available for analysis (i.e. $30 \%$ would be excluded owing to lack of space to introduce the IDB, the presence of diastema or the lack of teeth), the sample size increases to 914 sites $(=640 / 0.70)$. Considering 30 sites per participant, this results in a minimum sample size of 31 participants (i.e. approximately 16 participants per group).

To achieve the same sample size in both groups and simultaneously satisfy the randomization procedure to achieve balanced groups with respect to the most relevant variables (sex and baseline bleeding), a stratified (two levels for sex and two levels for baseline bleeding) block randomization (computer-assisted) method was used. Baseline bleeding was defined as the percentage of bleeding sites, per participant, as follows: a high level of bleeding if the participant had $\geq 30 \%$ bleeding sites; and a low level of bleeding if the participant had $<30 \%$ bleeding sites. Each participant was identified using a code. An allocation schedule was generated by computer by an independent assistant not involved in the study.

At baseline, the prescreened participants referred to the clinic for baseline examinations (gingivitis, periodontal conditions, and bleeding) had refrained from oral hygiene for at least $8 \mathrm{~h}$ but no more than $18 \mathrm{~h}$. A colorimetric probe (IAP Curaprox; Curaden, Kriens, Switzerland) was used to evaluate the diameter of the interproximal spaces, except those between the second and third molars (15), in order to determine the appropriate size of the IDB for each site. After the baseline oral examinations and 
assessments of other inclusion/exclusion criteria, qualifying participants were randomly assigned to one of two groups. Participants randomized to the control group brushed twice daily in their usual manner with an American Dental Association (ADA)-approved fluoride toothpaste and a soft-bristle toothbrush. Participants randomized to the active treatment group brushed twice daily in their usual manner, followed by interdental brushing once daily in the evening with their IDB formulation determined during the baseline examination with the colorimetric probe.

Participants in the test group received a manual soft-bristle toothbrush, an ADA-approved fluoride-containing dentifrice, and a pack of IDBs of different sizes (Curaprox CPS; Curaden). The first use of the product was conducted under the supervision of study personnel, following instruction of IDB use by a qualified public health professor. The instruction comprised verbal instructions on interdental brushing supported by practical demonstration on a plastic model. No further oral-hygiene instructions were provided. All other brushings were unsupervised, and the participants were required to maintain a diary card. Participants were instructed to mark the box corresponding to the current date on the diary card every evening after performing their interdental brushing to ensure that brushing was performed every day. Participants in the control group received an ADA-approved fluoride-containing dentifrice and a softbristle toothbrush at their baseline visit. No other oralhygiene procedures were permitted, including teeth cleaning and dental procedures. At baseline (T0), and at 1-wk (T1), 1-month $( \pm 2 \mathrm{~d})(\mathrm{T} 2)$, and 3-month (T3) visits, participants had brushed or used their IDBs at least $8 \mathrm{~h}$ previously but no more than $18 \mathrm{~h}$ previously. Two examiners collected the data at T0, T1, T2, and T3, and were blinded to the participant group allocation, which was assigned by an assistant not involved in the examination.

All participants were instructed in the modified Bass technique and were instructed to use a manual, compacttuft, soft-bristle toothbrush, twice a day. Participants in the test group were instructed with the appropriate IDB sizes determined at baseline and to introduce the calibrated IDBs once per day using different sizes for different sites.

At each visit during the evaluation period, the colorimetric probe was used in all interdental spaces for all participants in both groups. The procedure consists of introducing the colorimetric probe into the vestibular interdental space, inserting it fully, and then noting the colour that appears in the interdental space on the vestibular side. This colour corresponds to the colour of the IDB that is most suitable for the space in question. The probing protocol was always the same, starting in the 16-17 interdental space and finishing in the 46-47 interdental space. Information concerning the IDB diameter for each interdental space was recorded in a chart, and a copy was given to the participant. After horizontal probing, the corresponding brush was introduced into the interproximal space, and the presence of bleeding was observed. The pressure applied by a horizontal brush in the interdental area should be firm and continuous until reaching maximum compression with minimal discomfort to the patient. A visual analogue scale (VAS) was used to estimate the correlation between the patient's discomfort perception and the interproximal pressure $(0=$ no pain, $10=$ unbearable pain) (16). The pressure used to place the IDB was approximately $50-100 \mathrm{~N} \mathrm{~cm}^{-2}(0.20-0.40$ gram-force $)$, and $80 \%$ of participants were assigned a VAS score of $\leq 1$.
The IDBs used are from the CPS range of Curaprox (Curaden) products. This pack comprises five cylindrical IDBs with the following characteristics: a colour code related to the size of the brush; an access diameter defined by the gauge of the Cural (Curaden) wire core used to stiffen the IDB; and an effective cleaning diameter defined by the length of the synthetic bristles covering the working part of the device.

Two experienced examiners with graduate training in periodontics were trained beforehand in the use of the IAP Curaprox colorimetric probe (Curaden) and had obtained a minimum kappa value of 0.82 compared with the gold standard examiner (excellent agreement according to the LANDIS \& KocH scale (17). These examiners were trained in the use of the CPS Curaprox IDBs in a single cleaning movement, comprising three stages: (i) place an IDB at the point where the interdental gap begins, tilt the tip at an angle to the centreline of the teeth, and push it in gently at the correct angle as far as the centre of the teeth; (ii) without putting any more pressure on the brush, hold the handle at $90^{\circ}$ to the teeth; and (iii) push the brush through with care and then pull it out. The VAS was used (16). Examiners were blinded to each other, and the two observations were collected at an interval of at least $15 \mathrm{~min}$. The kappa statistic for the reproducibility of the pressure was $0.76(95 \% \mathrm{CI}: 0.14-1.38 ; P=0.02)$.

\section{Measures}

The Bleeding on Interdental Brushing Index (BOIB) was recorded, as was the bleeding response to the horizontal pressure applied in the interdental area by a calibrated IDB (18). After $30 \mathrm{~s}$, bleeding at each gingival unit was recorded according to the following scale: 0 , absence of bleeding after $30 \mathrm{~s}$; and 1, bleeding after $30 \mathrm{~s}$ (19).

\section{Statistical analysis}

SPSS Windows 20.0 (IBM, Chicago, IL, USA) was used for the descriptive statistics (mean values with SD and percentages) and for the analytical statistics ( $P$-value calculation) in those analyses in which the patient was the unit of analysis. SUDAAN 7.5 (RTI International, Research Triangle Park, NC, USA) was used for the analytical statistics ( $P$-value calculation) in those analyses in which the interproximal site was the unit of analysis to adjust for clustering (multiple sites within the patients). The output variable is the interproximal bleeding after IDB use at the interproximal site level. The statistical methods are indicated in the Table footnotes.

\section{Results}

All 46 randomized participants completed the study. The baseline characteristics of the intervention and control groups are presented in Table 1. The groups were broadly similar in terms of the principal variables at baseline, suggesting a high level of homogeneity between these two groups.

Table 2 shows the evolution of bleeding during the trial period. At T0, the percentage of bleeding sites was $34.8 \%$ in the control group and $35.9 \%$ in the test 
group $(P=0.88)$. During T0-T3, the evolution of the percentage of bleeding sites was not significant in the control group $(34.8 \%$ at $\mathrm{T} 0$ and $37.6 \%$ at T3;

Table 1

Baseline characteristics of trial participants according to study groups $(\mathrm{n}=46)$

\begin{tabular}{lccc}
\hline Variable & $\begin{array}{c}\text { Control } \\
(n=23)\end{array}$ & $\begin{array}{c}\text { Test } \\
(n=23)\end{array}$ & $P$ \\
\hline Sex & & & \\
$\quad$ Male & $16(69.6)$ & $14(60.9)$ & $0.757^{*}$ \\
$\quad$ Female & $7(30.4)$ & $9(39.1)$ & $0.409^{\dagger}$ \\
Age (yrs) & $22.8 \pm 3.8$ & $22.0 \pm 1.8$ & $0.768^{*}$ \\
Bleeding risk* & & & \\
$\quad$ High & $12(52.2)$ & $10(43.5)$ & $0.314^{*}$ \\
$\quad$ Low & $11(47.8)$ & $13(56.5)$ & \\
Smoker & & & \\
$\quad$ Yes & $8(34.8)$ & $19(82.6)$ & \\
$\quad$ No & $15(65.2)$ & &
\end{tabular}

Values are given as $n(\%)$ or mean $\pm \mathrm{SD}$.

*Chi-square, with Yates' correction.

†Student's $t$-test.

"High if $\geq 30 \%$ bleeding sites, and low if $<30 \%$ bleeding sites.
$P=0.10)$. In the test group, the frequency of bleeding sites decreased from $35.9 \%$ at T0 to $14.6 \%$ at T1, to $10.9 \%$ at $\mathrm{T} 2$, and to $10.4 \%$ at T3 $(P=0.008)$. The preventive fraction $(\mathrm{PF})$ was $46 \%$ at $\mathrm{T} 1,64 \%$ at $\mathrm{T} 2$, and $72 \%$ at $\mathrm{T} 3$.

Analysis of the PF according to localization (anterior sites or posterior sites) showed better effectiveness results for anterior sites. The PF values in anterior sites were $80 \%$ and $69 \%$ in the posterior sites at 3 months.

Table 3 shows the results of multilevel logistic regression analysis of the associations between candidate predictor variables and the presence of bleeding in a site after 3 months of interdental brushing. The OR for the presence of bleeding in the control group was 4.3. The interdental brushing had poorer results in participants with high baseline bleeding than in participants with low baseline bleeding $(\mathrm{OR}=2.3)$. The odds of bleeding were higher in posterior sites than in anterior sites $(\mathrm{OR}=2.2)$. Finally, we found an inverse relationship between the diameter of the IDB and the presence of bleeding: a larger diameter was associated with a smaller amount of bleeding.

Table 2

Bleeding frequency, according to study time point, for sites (in anterior and posterior regions) in participants in test $(\mathrm{n}=23)$ and control $(\mathrm{n}=23)$ groups

\begin{tabular}{|c|c|c|c|c|c|c|}
\hline \multirow[b]{2}{*}{ Zone and study time point } & \multicolumn{2}{|c|}{ Control } & \multicolumn{2}{|c|}{ Test } & \multirow{2}{*}{$\begin{array}{c}\text { Comparison } \\
(P)^{*}\end{array}$} & \multirow{2}{*}{$\begin{array}{c}\% \text { Difference } \\
\text { (effectiveness) } \\
(95 \% \mathrm{CI})^{\S}\end{array}$} \\
\hline & $n$ (sites) $^{*}$ & $\% \pm \mathrm{SE}^{\dagger}$ & $n$ (sites) & $\% \pm \mathrm{SE}$ & & \\
\hline \multicolumn{7}{|l|}{ Anterior } \\
\hline T0 (Baseline) & 170 & $28.2 \pm 6.2$ & 188 & $28.7 \pm 6.4$ & 0.956 & \\
\hline T1 (+1 wk from T0) & 170 & $17.6 \pm 6.1$ & 188 & $10.6 \pm 3.8$ & 0.335 & \\
\hline $\mathrm{T} 2$ (+1 month from $\mathrm{T} 0)$ & 170 & $28.8 \pm 6.4$ & 188 & $8.0 \pm 3.0$ & 0.005 & $72(48-96)$ \\
\hline T3 (+3 months from T0) & 170 & $29.4 \pm 6.4$ & 188 & $5.9 \pm 2.9$ & 0.001 & $80(59-100)$ \\
\hline Global $P^{*}$ & & 0.057 & & 0.019 & & \\
\hline Pairwise comparison** & & & & $\mathrm{T} 0 \neq \mathrm{T} 1, \mathrm{~T} 2, \mathrm{~T} 3$ & & \\
\hline \multicolumn{7}{|l|}{ Posterior } \\
\hline T0 (Baseline) & 370 & $37.8 \pm 4.9$ & 380 & $39.5 \pm 6.5$ & 0.841 & \\
\hline T1 (+1 wk from $\mathrm{T} 0)$ & 370 & $31.1 \pm 4.7$ & 380 & $16.6 \pm 4.2$ & 0.026 & $47(15-78)$ \\
\hline T2 (+1 month from T0) & 370 & $30.8 \pm 4.8$ & 380 & $12.4 \pm 3.0$ & 0.002 & $60(37-83)$ \\
\hline T3 (+3 months from T0) & 370 & $41.4 \pm 6.2$ & 380 & $12.6 \pm 5.3$ & 0.001 & $69(43-96)$ \\
\hline Global $P$ & & 0.227 & & 0.007 & & \\
\hline Pairwise comparison & & & & $\mathrm{T} 0 \neq \mathrm{T} 1, \mathrm{~T} 2, \mathrm{~T} 3$ & & \\
\hline \multicolumn{7}{|l|}{ Anterior + Posterior } \\
\hline T0 (Baseline) & 540 & $34.8 \pm 4.5$ & 568 & $35.9 \pm 6.2$ & 0.886 & \\
\hline T1 (+1 wk from T0) & 540 & $26.9 \pm 4.1$ & 568 & $14.6 \pm 3.6$ & 0.028 & $46(15-76)$ \\
\hline T2 (+1 month from T0) & 540 & $30.2 \pm 4.7$ & 568 & $10.9 \pm 2.5$ & $<0.001$ & $64(44-84)$ \\
\hline T3 (+3 months from T0) & 540 & $37.6 \pm 5.8$ & 568 & $10.4 \pm 4.2$ & $<0.001$ & $72(49-96)$ \\
\hline Global $P$ & & 0.102 & & 0.008 & & \\
\hline Pairwise comparison & & & & $\mathrm{T} 0 \neq \mathrm{T} 1, \mathrm{~T} 2, \mathrm{~T} 3$ & & \\
\hline
\end{tabular}

*Effective sample size for each estimation. For example, the first number value $(n=170)$ is obtained from the following calculation: 23 control patients $\times 10$ anterior sites/patient $=230$ sites, minus sites with diastemas $(n=12)$, lack of tooth $(n=7)$ or with no space to introduce the interproximal brush along the follow-up $(n=41)$, gives effective sample $=170$ sites (=230 minus 12 minus 7 minus 41$)$.

${ }^{\dagger}$ Standard errors (SEs) corrected for complex sampling (multiple sites within the mouth), using the DESCRIPT procedure in SUDAAN 7.0.

*Values of $P$ corrected for complex sampling (multiple sites within the mouth), using chi-square analysis (CROSSTAB procedure in SUDAAN 7.0).

${ }^{\S}$ Per cent difference between control $(C)$ and test $(T)$ subjects $=\{[(\% C-\% T) / \% C] \times 100\}$.

$995 \%$ CI $=\% \pm 1.96 \mathrm{SE}$, where the standard error (SE) is calculated, after correction for multiple sites within the mouth (using the DESCRIPT procedure in SUDAAN 7.0), according to DUBEY et al. (14).

**When the global $P$-value is significant, paired comparisons were performed using chi-square analysis, corrected for complex sampling; the symbol ' $\neq$ ' indicates significantly $(P<0.05)$ different groups. 
Table 3

Multivariate associations* between studied variables and bleeding at 3 months after interproximal brushing $\left(\mathrm{n}=1,108^{\dagger}\right.$ sites from 46 subjects)

\begin{tabular}{lllc}
\hline Variable & $n$ & OR $(95 \% \mathrm{CI})$ & $P$ \\
\hline $\begin{array}{l}\text { Participant-level variables } \\
\text { Group }\end{array}$ & & & \\
$\quad$ Control (no interproximal & 540 & $4.3(1.6-12.1)$ & 0.006 \\
$\quad$ brushing) & & & \\
$\quad$ Test (interproximal brushing) & 568 & 1 & \\
Baseline bleeding risk & & & \\
$\quad$ High & 526 & $2.3(0.9-5.5)$ & 0.065 \\
$\quad$ Low & 582 & 1 & \\
Site-level variables & & & \\
Zone & 750 & $2.2(1.5-3.3)$ & $<0.001$ \\
Posterior & 358 & 1 & \\
Anterior & & & \\
Interproximal brush at 3 months & 112 & $0.0(0.0-0.2)$ & 0.005 \\
$\quad 5(1.1 \mathrm{~mm})$ & 159 & $0.1(0.0-0.5)$ & \\
$\quad 4(0.9 \mathrm{~mm})$ & 251 & $0.5(0.3-1.1)$ & \\
$3 \quad(0.8 \mathrm{~mm})$ & 405 & $0.6(0.3-1.0)$ & \\
$2(0.7 \mathrm{~mm})$ & 181 & 1 & \\
$1(0.6 \mathrm{~mm})$ & &
\end{tabular}

*Backward stepwise based on statistical significance $(P>0.15$ to exclude a variable). Initial variables included also age, sex, and smoking status. Values of $P$ and $95 \%$ CI were calculated using LOGISTIC PROC in SUDAAN 7.0, to account for clustering (multiple sites within patients).

These data refer to 46 patients $\times 30$ sites $/$ patient $=1,380$ sites; sites were excluded owing to presence of diasthema $(n=21)$, lack of tooth $(n=163)$, or lack of space to introduce the interproximal brush during the follow up $(n=80)$; thus, available sites for this table $=1,167$.

\section{Discussion}

To the best of our knowledge, the present study is the first randomized clinical trial based on the CONSORT statement $(11,20)$ that describes the efficiency of interdental calibrated brushes on interproximal bleeding in healthy periodontal adults.

The first objective of our study was to examine whether CPS interdental calibrated brushes are effective in reducing the frequency of interproximal bleeding when used with regular toothbrushing. A potential limitation of this study was the absence of a group using dental floss or other IDBs. In the literature, the quality of the only published study comparing toothbrushing plus interdental brushing with toothbrushing alone is considered low (3). Only one study, which had a high risk of bias, has compared interdental brushing and toothbrushing with toothbrushing alone (21). There is no agreed-upon scientific method for choosing IDBs (5). The so-called reference technique is the empirical subjective method in everyday use. This technique consists of testing the various IDBs in order of increasing diameter. The use of colorimetric probes and interdental brushing in our study was more beneficial than interdental brushing alone and can be considered a new technique for increasing the concordance between the empirical choice of IDBs of different diameters and the gold standard (5).
This explanatory randomized control study provide information on whether the intervention is effective under optimal conditions, including strict eligibility criteria, compliant subjects, maximum monitoring of practitioner adherence to the study protocol, and formal follow-up visits (22). With respect to such criteria, volunteers of the Faculty of Oral Medicine University differ from many other groups in society in a number of ways, which has implications for the generalizability of the results (external validity). However, the quality of the results in an explanatory approach is not affected.

Regarding the sampling method, there are several important points to consider. First, the analysis is performed on all interdental sites using the SUDAAN program, which allows for adjustment of the $P$-values and standard errors from clustering (i.e. multiple sites within the mouth). Second, to achieve correct randomization in the two groups, balanced selection was used in terms of sex and baseline bleeding, as described in the Material and Methods. Therefore, the allocation has been made without any bias. Bleeding on IBD pressure was used as the dependent variable. According to LORENZ et al., there is no doubt that indices containing a bleeding component can successfully be used in clinical trials (23). However, the reproducibility of the diagnosis of bleeding has been widely discussed in the literature (24). The force applied to the IDB, the angle of insertion, and the experience of the examiner are cited as factors of misdiagnosis. The bleeding index can also be influenced by the initial oral hygiene standard of the participants (25). Therefore, participants were stratified according to the baseline levels of bleeding to ensure equal distribution.

The internal validity of the study was guaranteed using a calibration process. The two examiners who participated in data collection were previously trained in a calibration workshop. The same examiners had participated in a previous study using a similar protocol. The blinding of the examiners was guaranteed throughout the study. None of the participants in either group dropped out of the study, which was certainly because of the specific characteristics of the sample.

An interesting issue is the association of bleeding with the need for periodontal care in the medium and long term. Gingivitis is a reversible condition associated with bacterial biofilms; it generally resolves clinically within approximately $1 \mathrm{wk}$ after the reinstitution of oral-hygiene procedures (26). It could be easily argued that the natural occurrence of gingival inflammation describes a condition whose characteristics are normally distributed in the population (27). The reduction of gingivitis in the general population results in more than merely cosmetic improvement following the reduction of gingival bleeding. However, there is overwhelming evidence that gingivitis is linked to periodontitis, and the elimination of gingivitis will result in the reduction of attachment loss in the majority of the population (28).

Adherence to daily dental flossing is low among patients because it requires a certain degree of dexterity 
and motivation (29); patients prefer IDBs because they are easier to use $(4,30)$. Furthermore, when compared with dental floss, IDBs are thought to be more effective for plaque removal because the bristles fill the embrasure and are able to clean the invaginated areas on the tooth and root surfaces (9). In addition, dental floss does not reach the anatomical concavities of the proximal surfaces of premolars and molars, which renders the flossing effort incomplete (31).

Our findings suggest a positive impact of the use of IDBs in reducing interproximal bleeding of periodontally healthy young participants. From week 1, the observed reduction was $47 \%$ compared with the control. At 3 months, bleeding was reduced by $71 \%$. No undesired side effects from using IDBs were observed over the 3-month study period. These findings need to be confirmed over a longer time period and the intervention should be compared, in clinical trials, with other methods of interdental brushing, such as dental floss and dental sticks of different kinds.

The study findings offer evidence of the effectiveness of a programme for reducing bleeding through daily IDB use in young adults. The attendance rate for the intervention sessions was high because the participants were from the Faculty of Oral Medicine. While these results may support the feasibility of a population approach to promoting dental health through interdental brushing coaching programmes, it is still necessary to evaluate the effectiveness of the procedure in real-life conditions.

Conflict of interest - The authors declare that they have no conflicts of interest related to this study.

\section{References}

1. Chandki R, Banthia P, Banthia R. Biofilms: a microbial home. J Indian Soc Periodontol 2011; 15: 111-114.

2. Slot DE, Dörfer CE, van der Weijden GA. The efficacy of interdental brushes on plaque and parameters of periodontal inflammation: a systematic review. Int J Dent Hyg 2008; 6: 253-264.

3. Poklepovic T, Worthington HV, Johnson TM, Sambungak D, Imai P, Clarkson JE, Tugwell P. Interdental brushing for the prevention and control of periodontal diseases and dental caries in adults. Cochrane Database Syst Rev 2013; 12: CD009857.

4. Imai PH, Hatzimanolakis PC. Encouraging client compliance for interdental care with the interdental brush: the client's perspective. Can J Dent Hyg 2010; 44: 56-60.

5. Bourgeois D, Carrouel F, Llodra JC, Bravo M, Viennot S. A colorimetric interdental probe as a standard method to evaluate interdental efficiency of interdental brush. Open Dent J 2015; 9: 431-437.

6. Claydon NC. Current concepts in tooth brushing and interdental cleaning. Periodontol 2000 2008; 48: 10-22.

7. Knöfler GU, Purschwitz RE, Eick S, Pfister W, Roedel M, Jentsch HF. Microbiologic findings 1 year after partialand full-mouth scaling in the treatment of moderate chronic periodontitis. Quintessence Int 2012; 43: e23-e31.

8. Noorlin I, Watts TL. A comparison of the efficacy and ease of use of dental floss and interproximal brushes in a randomized split mouth trial incorporating an assessment of subgingival plaque. Oral Health Prev Dent 2007; 5: 13-18.
9. Imai PH, Hatzimanolakis PC. Interdental brush in type I embrasures: examiner blinded randomized clinical trial of bleeding and plaque efficacy. Can J Dent Hyg 2011; 45: 13-20.

10. Berchier CE, Slot DE, Haps S, van der Weijden GA. The efficacy of dental floss in addition to a toothbrush on plaque and parameters of gingival inflammation: a systematic review. Int J Dent Hyg 2008; 6: 265-279.

11. Moher D, Schulz KF, Altman DG. The CONSORT statement: revised recommendations for improving the quality of reports of parallel group randomized trials. BMC Med Res Methodol 2001; 1: 2.

12. Armitage GC. Development of a classification system for periodontal diseases and conditions. Ann Periodontol 1999; 4: 1-6.

13. da Silva-Boghossian CM, do Souto RM, Luiz RR, Colombo AP. Association of red complex, A. actinomycetemcomitans and non-oral bacteria with periodontal diseases. Arch Oral Biol 2011; 56: 899-906.

14. Dubey SO, Lehnhoff RW, Radike AW. A statistical confidence interval for true percent reduction in caries incidence studies. J Dent Res 1965; 44: 921-923.

15. Palmer RM, Wilson RF, Hasan AS, Scott DA. Mechanisms of action of environmental factors-tobacco smoking. $J$ Clin Periodontol 2005; 32(Suppl 6): 180-195.

16. Checchi L, Montevecchi M, Marucci G, Checchi V. A proposed new index for clinical evaluation of interproximal soft tissues: the interdental pressure index. Int J Dent 2014; 114: 345075.

17. Landis JR, Koch GG. The measurement of observer agreement for categorical data. Biometrics 1977; 33: 159-174.

18. Caton JG, Polson AM. The interdental bleeding index: a simplified procedure for monitoring gingival health. Compend Contin Educ Dent 1985; 6: 88-92.

19. Hofer D, Sahrmann P, Attin T, Schmidlin PR. Comparison of marginal bleeding using a periodontal probe or an interdental brush as indicators of gingivitis. Int J Dent Hyg 2011; 9: 211-215.

20. Geminiani A, Ercoli C, Feng C, Caton JG. Bibliometrics study on authorship trends in periodontal literature from 1995 to 2010. J Periodontol 2014; 85: e136-e143.

21. Jared H, Zhong Y, Rowe M, Ebisutani K, Tanaka T, TAKASE N. Clinical trial of a novel interdental brush cleaning system. J Clin Dent 2005; 16: 47-52.

22. Chan AW, Tetzlaff JM, Gøtzsche PC, Altman DG, Mann H, Berlin JA, Dickersin K, Hróbjartsson A, Schulz KF, Parulekar Wr, Krleža-Jerić K, Laupacis A, Moher D. SPIRIT 2013 explanation and elaboration: guidance for protocols of clinical trials. BMJ 2013; 346: e7586.

23. Lorenz K, Bruhn G, Netuschil L, Heumann C, Hoffmann T. How to select study designs and parameters to investigate the effect of mouthrinses? Part I: rationale and background. J Physiol Pharmacol 2009; 60(Suppl 8): 77-83.

24. Leroy R, Eaton K, Savage A. Methodological issues in epidemiological studies of periodontitis- how can be improved? BMC Oral Health 2010; 10: 1-8.

25. Kossack C, Jost-Brinkmann PG. Plaque and gingivitis reduction in patients undergoing orthodontic treatment with fixed appliances-comparison of toothbrushes and interdental cleaning aids. A 6-month clinical single-blind trial. J Orofac Orthop 2005; 66: 20-38.

26. Pihlstrom BL, Michalowicz BS, Johnson NW. Periodontal diseases. Lancet 2005; 366: 1809-1820.

27. Mariotti A, Hefti AF. Defining periodontal health. $B M C$ Oral Health 2015; 15(Suppl 1): S6.

28. Robinson PJ. Gingivitis: a prelude to periodontitis? J Clin Dent 1995; 6(Spec issue): 41-45.

29. Asadoorian J, Locker D. The impact of quality assurance programming: a comparison of two Canadian dental hygienist programs. J Dent Educ 2006; 70: 965-971.

30. Christou V, Timmerman MF, van der Velden U, van der WeIJden FA. Comparison of different approaches of interdental oral hygiene: interdental brushes versus dental floss. J Periodontol 1998; 69: 759-764.

31. Jackson MA, Kellett M, Worthington HV, Clerehugh V. Comparison of interdental cleaning methods: a randomized controlled trial. $J$ Periodontol 2006; 77 : 1421-1429. 